\title{
Preoperative Frailty and Quality of Recovery After Neoplastic Curative Surgery
}

Azevedo J.', Sousa M.', Carvalho B.', Marinho R.', Santos A.', Abelha F.',2 'Centro Hospitalar de São João, Dept of Anaesthesiology, Porto, Portugal ${ }^{2}$ Faculdade de Medicina da Universidade do Porto, Portugal

\section{Background and Goal of Study}

The concept of frailty describes a state of increased vulnerability and is a significant predictor of adverse outcomes. We aim to evaluate the incidence of frailty in patients with neoplastic disease and the impact of this condition in postoperative recovery.

\section{Materials and Methods}

Prospective and observational study;

Inclusion criteria: adult patients submitted to elective curative neoplastic surgery and admitted to the Post Anaesthetic Care Unit;

- Exclusion criteria: inability to give informed consent; Frail patients (FP): Clinical Frailty Scale score $\geq 4$;

Quality of life before surgery:

EuroQol Five Dimensions Questionnaire (EQ-5D).

Postoperative recovery:

Postoperative Quality of Recovery Scale (PQRS) at baseline and after surgery at minute I5 (TI5), 40 (T40) and days I(DI) and 3 (D3);

Quality of Recovery I5 (QoR- I5) at baseline (D0) and day I after surgery (DI).

> Poor Quality of Recovery (PQR) was considered using a calculation (mean DI QoR-I5 score minus I SD);

> The Mann-Whitney test, Chi-square or Fisher's exact test were used. Statistical significance when $p<0,05$.

\section{Results}

Of I 48 patients, $30 \%$ were frail.

Table I - Frailty and Cardiac Risk profile Univariate analysis

\begin{tabular}{|c|c|c|c|}
\hline Variables & $\begin{array}{l}\text { Non Frail } \\
N=103\end{array}$ & Frail $N=45$ & P value \\
\hline Age, median [P25-P75 & $\begin{array}{c}62,0 \\
{[51,0-70,0]}\end{array}$ & $\begin{array}{c}73,0 \\
{[66,0-78,0]}\end{array}$ & $<0,\left.00\right|^{a}$ \\
\hline Male gender, $\mathrm{N}(\%)$ & $49(47,6)$ & $16(35,6)$ & $0,209^{b}$ \\
\hline ASA $\geq \mathrm{III}, \mathrm{n}(\%)$ & $23(22,3 \%)$ & $28(62,2 \%)$ & $<0,\left.00\right|^{c}$ \\
\hline $\begin{array}{l}\text { High-risk procedures, } \\
\text { N (\%) }\end{array}$ & $40(38,8)$ & $19(31,1)$ & $0.699^{c}$ \\
\hline $\begin{array}{l}\text { History of Ischemic } \\
\text { Heart Disease, N (\%) }\end{array}$ & $7(6,8)$ & $14(31,1)$ & $<0,\left.00\right|^{c}$ \\
\hline $\begin{array}{l}\text { History of Heart Failure, } \\
\qquad \mathrm{N}(\%)\end{array}$ & $6(5,8)$ & II $(24,4)$ & $0,00 I^{c}$ \\
\hline $\begin{array}{c}\text { Insulin dependent } \\
\text { Diabetes mellitus, } \mathrm{N}(\%)\end{array}$ & $2(1,9)$ & $6(13,3)$ & $0,00 I^{b}$ \\
\hline $\begin{array}{c}\text { Preoperative serum } \\
\text { creatinine } \geq 2.0 \mathrm{mg} / \mathrm{dL}, \mathrm{N}(\%)\end{array}$ & $2(1,9)$ & $6(13,3)$ & $0,0 I^{b}$ \\
\hline $\begin{array}{l}\text { History of Cerebrovascular } \\
\text { Disease, } N(\%)\end{array}$ & $2(1,9)$ & $2(4,4)$ & $0,355^{\mathrm{b}}$ \\
\hline $\mathrm{RCRI} \leq 2, \mathrm{~N}(\%)$ & $93(90.3)$ & $30(66.7)$ & \multirow[t]{2}{*}{$<0,\left.00\right|^{c}$} \\
\hline $\mathrm{RCRI}>2, \mathrm{~N}(\%)$ & $10(9.7)$ & $15(33.3)$ & \\
\hline
\end{tabular}

Table 2 - Quality of Life before surgery

\begin{tabular}{|c|c|c|c|}
\hline $\begin{array}{c}\text { EQ-5D } \\
\text { (problems/limitations) }\end{array}$ & $\begin{array}{c}\text { Non } \\
\text { Frail } \\
\text { N= } 103\end{array}$ & $\begin{array}{l}\text { Frail } \\
N=45\end{array}$ & P Value \\
\hline Mobility, N (\%) & II $(10,7)$ & $33(73.3)$ & $<0.00 I^{\mathrm{C}}$ \\
\hline Self-care, N (\%) & $2(1.9)$ & $21(46.7)$ & $<\left.0.00\right|^{c}$ \\
\hline Usual activities, $\mathrm{N}(\%)$ & II (I0.7) & 32 (7I.I) & $<0.00 I^{c}$ \\
\hline Pain/Discomfort, N (\%) & $25(24.3)$ & $28(62.2)$ & $<\left.0.00\right|^{c}$ \\
\hline Anxiety/Depression, N (\%) & 80 (77.7) & 37 (82.2) & $0.53 I^{c}$ \\
\hline $\begin{array}{l}\text { Health status, self evaluation, } \\
\text { median (P25-P-75) }\end{array}$ & $\begin{array}{c}70 \\
{[50-80]}\end{array}$ & $\begin{array}{c}50 \\
{[40-65]}\end{array}$ & $<0,\left.00\right|^{a}$ \\
\hline
\end{tabular}

Table 3 - PQRS Incomplete recovery Univariate analysis

\begin{tabular}{|c|c|c|c|c|}
\hline \multicolumn{2}{|c|}{$\begin{array}{c}\text { PQRS Incomplete } \\
\text { Recovery } \\
\text { (domain/time frame) }\end{array}$} & \multirow{2}{*}{$\begin{array}{l}\text { Non Frail } \\
\text { N= } 103 \\
88(85,4)\end{array}$} & \multirow{2}{*}{$\begin{array}{l}\text { Frail } N=45 \\
42(93,3)\end{array}$} & \multirow{2}{*}{$\begin{array}{l}\text { P value } \\
0,274^{b}\end{array}$} \\
\hline \multirow{3}{*}{$\begin{array}{c}\text { Physiologic, } \\
\text { N (\%) }\end{array}$} & TI5 & & & \\
\hline & T40 & $70(67,9)$ & $36(80,0)$ & $0,10 I^{c}$ \\
\hline & DI & $20(19,4)$ & $18(40,0)$ & $0,0 \mid 4^{c}$ \\
\hline \multirow{4}{*}{$\begin{array}{c}\text { Nociceptive, } \\
\text { N (\%) }\end{array}$} & TI5 & $17(16,5)$ & $18(40,0)$ & $0,002^{c}$ \\
\hline & T40 & $23(22,3)$ & $19(42,2)$ & $0,0 / 2^{c}$ \\
\hline & DI & $21(20,4)$ & $17(37,8)$ & $0,\left.02\right|^{c}$ \\
\hline & D3 & $17(16,5)$ & $19(42,2)$ & $<0,\left.00\right|^{c}$ \\
\hline \multirow{4}{*}{$\begin{array}{c}\text { Emotional, } \\
\text { N (\%) }\end{array}$} & TI5 & $64(62,1)$ & $34(75,5)$ & $0,112^{c}$ \\
\hline & T40 & $65(63, I)$ & $32(7 I, I)$ & $0,\left.29\right|^{c}$ \\
\hline & DI & $57(55,3)$ & $30(66,6)$ & $0,15 I^{c}$ \\
\hline & D3 & $57(55,3)$ & $27(60,0)$ & $0,363^{c}$ \\
\hline \multirow{4}{*}{$\begin{array}{c}\text { Cognitive, } \\
\text { N (\%) }\end{array}$} & TI5 & $96(93,2)$ & $4 \mid(9 I, I)$ & $0,736^{b}$ \\
\hline & T40 & $88(85,4)$ & $35(77,8)$ & $0,54 I^{c}$ \\
\hline & DI & $81(78,6)$ & $35(77,8)$ & $0,956^{c}$ \\
\hline & D3 & $77(74,8)$ & $34(75,5)$ & $0,967^{c}$ \\
\hline \multirow{3}{*}{$\begin{array}{c}\text { Functional, } \\
\text { N (\%) }\end{array}$} & T40 & $82(79,6)$ & $37(82,2)$ & $0,597^{c}$ \\
\hline & DI & $66(64, I)$ & $30(66,7)$ & $0,936^{c}$ \\
\hline & D3 & $52(50,5)$ & $23(5 \mathrm{I}, \mathrm{I})$ & $0,853^{c}$ \\
\hline
\end{tabular}

Table 4 - QoR- 15 and PQR Univariate analysis

\begin{tabular}{|c|c|c|c|}
\hline & $\begin{array}{c}\text { Non Frail } \\
N=103\end{array}$ & Frail $N=45$ & P Value \\
\hline $\begin{array}{c}\text { QoR-I } 5 \text { D0, median } \\
\text { [P25-P75] }\end{array}$ & $\begin{array}{c}133.5 \\
{[|24-| 4 \mid]}\end{array}$ & $\begin{array}{c}\mid 14 \\
{[96.5-\mid 28.5]}\end{array}$ & $<0,\left.00\right|^{a}$ \\
\hline $\begin{array}{l}\text { QoR-I5 DImedian } \\
\text { [P25-P75] }\end{array}$ & $\begin{array}{c}115 \\
{[101.3-127]}\end{array}$ & $\begin{array}{c}I|| \\
{[87.8-125.5]}\end{array}$ & $0.155^{a}$ \\
\hline PQR, N (\%) & II (10.7) & II (24.4) & $0.030^{c}$ \\
\hline
\end{tabular}

a: Mann-Whitney $U$ test test; b: Fisher's exact test; c: chi-squared test

\section{Conclusion}

The incidence of frailty was $30 \%$.

FP were older, had higher RCRI and worst health status prior surgery.

QoR-I5 identified PQR in frail neoplastic patients and PQRS revealed incomplete recovery in physiologic domain at $\mathrm{DI}$ and in nociceptive domain at all time frames 